Georgian Mathematical Journal

1(1994), No. 5, 505-521

\title{
LINEAR DYNAMICAL SYSTEMS OF HIGHER GENUS
}

\author{
V. LOMADZE
}

\begin{abstract}
A class of linear systems which after ordinary linear systems are in a certain sense the simplest ones, is associated with every algebraic function field. From the standpoint developed in the paper ordinary linear systems are associated with the rational function field.
\end{abstract}

\section{$\S 0$. InTRODUCTION.}

As is well known, there is a close relationship between linear systems and the rational function field. The subject of the paper is to study new linear systems which are closely connected with arbitrary algebraic function fields.

The idea of introducing linear systems of "higher genus" is due to R.Hermann [7]. He tries to describe them in terms of linear spaces of infinite dimension. Our approach is different and uses vector bundles (of finite rank) over algebraic function fields.

In what follows we shall assume that the reader is familiar with the elementary concepts of commutative algebra such as a discrete valuation, a Dedekind domain, a maximal ideal, an exact sequence of modules and a localization. In the appendix we give all necessary concepts and facts from the theory of algebraic function fields.

Throughout the paper, $k$ will denote a ground field, and $m$ and $p$ input and output numbers, respectively. We fix once and for all:

an algebraic function field $R$ over $k$;

a discrete valuation $v$ of $R$ trivial on $k$ and such that its residue field coincides with $k$;

a function $s$ such that $v(s)=-1$.

The simplest example $(\star)$ is given by the following data:

$R=k(z)$ where $z$ is an indeterminate;

$v=$ discrete valuation determined by the formula $v(f / g)=\operatorname{deg} g-\operatorname{deg} f$

if $f, g \in k[z], g \neq 0$

$s=z$.

1991 Mathematics Subject Classification. 93A99, 93B05, 93B07, 93B25, 93 B55.

505

1072-947X/1994/0900-0505\$07.00/0 @ 1994 Plenum Publishing Corporation 
Let $X$ denote the set of all places of $R$. Recall that each place $x$ gives a discrete valuation $\operatorname{ord}_{x}$ of $R$ trivial on $k$, and that this correspondence is bijective. Denote by $\infty$ the place corresponding to $v$ and call it the infinite place. Let $\mathcal{O}$ denote the standard vector bundle over $R$. For any divisor $D$ the associated vector bundle is denoted by $\mathcal{O}(D)$. For each integer $n$ let us write $\mathcal{O}(n)$ instead of $\mathcal{O}(n \infty)$. Let $A$ denote the ring of functions which are regular outside from $\infty$. This is a Dedekind domain. Its maximal ideals are in the one-to-one correspondence with places distinct from $\infty$. Finally, let $K$ denote the divisor of the differential $d s$ and $g$ the genus of $R$.

We define a linear system over $(R, v, s)$ as a quintuple $(V, \mathcal{E}, \theta, B, C)$ consisting of a linear space $V$ over $k$, a vector bundle $\mathcal{E}$ over $R$, a morphism $\theta: \mathcal{O} \otimes V \rightarrow \mathcal{E}$ and linear maps $B: k^{m} \rightarrow H^{0} \mathcal{E}(-1), C: V \rightarrow k^{p}$. It is required that the following conditions hold:

(1) $H^{1} \mathcal{E}(K)=0$;

(2) $\theta$ induces a bijective linear map $V \rightarrow \mathcal{E}(\infty)$;

(3) the canonical map $H^{0} \mathcal{O}(K) \otimes V \oplus H^{0} \mathcal{O}(K)^{m} \rightarrow H^{0} \mathcal{E}(K)$ is surjective.

It is the goal of the paper to show that this definition should lead to an interesting theory.

Let us see what linear systems are in the example $(\star)$. We have: $K=$ $-2 \infty, H^{0} \mathcal{O}(-2)=H^{1} \mathcal{O}=0$. So the conditions (1) and (3) can be rewritten as $H^{1} \mathcal{E}(-2)=0$ and $H^{0} \mathcal{E}(-2)=0$, respectively. Vector bundles with the above properties and linear spaces (of finite dimension) are made equivalent by the functors $\mathcal{E} \rightarrow H^{0} \mathcal{E}(-1)$ and $W \rightarrow \mathcal{O}(1) \otimes W$. Next, giving a morphism $\theta: \mathcal{O} \otimes V \rightarrow \mathcal{O}(1) \otimes W$ is equivalent to giving a pair of linear maps $E, A: V \rightarrow W$. It follows that in the case of $(\star)$ a linear system can be described in terms of linear algebra, namely, as a sextuple $(V, W, E, A, B, C)$ where $V$ and $W$ are finite-dimensional linear spaces, $E: V \rightarrow W$ is a bijective linear map and $A: V \rightarrow W, B: k^{m} \rightarrow W, C: V \rightarrow k^{p}$ are arbitrary linear maps. It is easily seen that such sextuples are equivalent to ordinary linear systems. (The equivalence is established by

$$
\left.(V, W, A, E, B, C) \longrightarrow\left(V, E^{-1} A, E^{-1} B, C\right) .\right)
$$

Thus linear systems associated with $(\star)$ and ordinary linear systems are the same thing.

The paper is organized as follows.

In $\S 1$ we define controllability, observability, transfer functions and Martin-Hermann sheaves. Here we also introduce a category $\Sigma$ whose objects are triples $(\mathcal{F}, D, N)$ consisting of a coherent sheaf $\mathcal{F}$ generated by global sections, a morphism $D: \mathcal{O}^{m} \rightarrow \mathcal{F}$ such that $D(\infty): k^{m} \rightarrow \mathcal{F}(\infty)$ is a bijection and a morphism $N: \mathcal{O}^{p} \rightarrow \mathcal{F}$ such that $N(\infty): k^{p} \rightarrow \mathcal{F}(\infty)$ is zero.

In $\S 2$ we prove that the category of linear systems is equivalent to the opposite category of $\Sigma$. This means that a linear system can be defined as an 
object of $\Sigma .{ }^{1}$ From this we easily derive Kalman's theorem on realization.

In $\S 3$ we define a feedback equivalence and prove the Martin-Hermann theorem which says that two linear systems are feedback equivalent if and only if their Martin-Hermann sheaves are isomorphic. Then we discuss the pole-placement theorem. Unfortunately, we prove it for one input linear systems only.

\section{$\S 1$. Linear Systems}

In this section we do not impose the third condition on linear systems. So by a linear system here we shall mean a quintuple $(V, \mathcal{E}, \theta, B, C)$ where $V$ is a linear space, $\mathcal{E}$ is an effective vector bundle such that $H^{1} \mathcal{E}(K)=0, \theta$ is a morphism of $\mathcal{O} \otimes V$ into $\mathcal{E}$ such that the linear map $\theta(\infty): V \rightarrow \mathcal{E}(\infty)$ is bijective, $B$ is a linear map $k^{m} \rightarrow H^{0} \mathcal{E}(-1)$ and $C$ is a linear map $V \rightarrow k^{p}$.

1. Let $\sigma=(V, \mathcal{E}, \theta, B, C)$ be a linear system.

Definition. We define the rank of $\sigma$ as the rank of $\mathcal{E}$ or, what is the same, as the dimension of $V$. We define the McMillan degree of $\sigma$ as the degree of $\mathcal{E}$.

Definition. The characteristic sheaf of $\sigma$ is defined to be the cokernel of $\theta$.

If $\mathcal{C}$ denotes the characteristic sheaf of $\sigma$, then, by definition, one has an exact sequence

$$
0 \longrightarrow \mathcal{O} \otimes V \longrightarrow \mathcal{E} \longrightarrow \mathcal{C} \longrightarrow 0 .
$$

Definition. The state sheaf of $\sigma$ is defined to be $\mathcal{C}(K)$, and the pole sheaf of $\sigma$ is defined to be $\mathcal{E} x t^{1}(\mathcal{C}, \mathcal{O})$.

Observe that to give a linear map $k^{m} \rightarrow H^{0} \mathcal{E}(-1)$ is to give a morphism $\mathcal{O}^{m} \rightarrow \mathcal{E}$, which induces a zero map on the reduced stalks at infinity. (This follows from the exact sequence $0 \rightarrow H^{0} \mathcal{E}(-1) \rightarrow H^{0} \mathcal{E} \rightarrow \mathcal{E}(\infty)$.) Likewise, to give a linear map $V \rightarrow k^{p}$ is to give a morphism $\mathcal{O} \otimes V \rightarrow \mathcal{O}^{p}$. For this reason we shall use the same letters $B$ and $C$ for the corresponding morphisms.

One defines morphisms of linear systems in the obvious way.

2. Let $\sigma=(V, \mathcal{E}, \theta, B, C)$ be a linear system.

Definition. If $x$ is a finite place, then we say that

(a) $\sigma$ is reachable at $x$ if $\operatorname{rk}[\theta(x) B(x)]=\operatorname{dim} V$, i.e. if the morphism

$$
[\theta B]: \mathcal{O} \otimes V \oplus \mathcal{O}^{m} \longrightarrow \mathcal{E}
$$

\footnotetext{
${ }^{1}$ Such a definition was in fact the starting point of the paper. One immediately comes to it through Corollary 4 of Theorem 1 from [10].
} 
is surjective at $x$;

(b) $\sigma$ is observable at $x$ if

$$
\operatorname{rk}\left[\begin{array}{c}
\theta(x) \\
C
\end{array}\right]=\operatorname{dim} V
$$

i.e. if the morphism

$$
\left[\begin{array}{c}
\theta \\
C
\end{array}\right]: \mathcal{O} \otimes V \longrightarrow \mathcal{E} \oplus \mathcal{O}^{p}
$$

is left invertible at $x$.

Because $\theta$ is bijective at one place, namely at infinity, it should be bijective at all but finitely many places. This implies, in particular, that every linear system is reachable (observable) at all but finitely many places.

For each $N \geq 0$ put $\Omega(-N)=H^{0} \mathcal{O}(K+(N+2) \infty)$. We then have a composition series

$$
H^{0} \mathcal{O}(K) \subseteq \Omega(0) \subseteq \Omega(-1) \subseteq \Omega(-2) \subseteq \cdots .
$$

Let $\Omega=\cup \Omega(-N)$. $\Omega$ consists of the sections of $\mathcal{O}(K)$ over the affine open set $X-\{\infty\}$, and therefore, is a projective $A$-module of rank 1. All successive quotients in the above series are one dimensional linear spaces.

Example. For $(\star)$ we have: $H^{0} \mathcal{O}(K)=0$ and $\Omega(-N)=$ the space of polynomials in $s$ of degree $\leq N\}$. Therefore $\Omega=k[s]$.

Set $\Gamma=R / \Omega$. This is an injective $A$-module. We have

$$
R / H^{0} \mathcal{O}(K) \supseteq R / \Omega(0) \supseteq R / \Omega(-1) \supseteq R / \Omega(-2) \cdots
$$

and $\Gamma=\cap R / \Omega(-N)$.

Following R.Kalman we call $\Omega^{m}$ the input module and $\Gamma^{p}$ the output module.

Now let $\mathcal{C}, \mathcal{S}$ and $\mathcal{P}$ denote the characteristic, the state and the pole sheaves of $\sigma$, respectively. These three sheaves may be regarded as $A$ modules of finite length because their supports do not contain $\infty$.

Tensoring the morphism $[\theta B]$ by $\mathcal{O}(K)$, we obtain a morphism $\mathcal{O}(K) \otimes$ $V \oplus \mathcal{O}(K) \rightarrow \mathcal{E}(K)$. This gives a morphism $\mathcal{O}^{m}(K) \rightarrow \mathcal{S}$ and hence a homomorphism of $A$-modules

$$
I(\sigma): \Omega^{m} \longrightarrow \mathcal{S} .
$$

Further, dualizing $\left[\begin{array}{c}\theta(K) \\ C\end{array}\right]$, we obtain a morphism $\mathcal{E}^{*} \oplus \mathcal{O}^{p} \rightarrow \mathcal{O} \otimes V^{*}$. This gives a morphism $\mathcal{O}^{p} \rightarrow \mathcal{P}$ and hence a homomorphism of $A$-modules $A^{p} \rightarrow \mathcal{P}$. Applying now the functor $\operatorname{Hom}_{A}(\cdot, \Gamma)$, we get a homomorphism

$$
O(\sigma): \mathcal{S} \longrightarrow \Gamma^{p} .
$$


We call $I(\sigma)$ the input homomorphism and $O(\sigma)$ the output homomorphism of $\sigma$.

Proposition 1. Let $\sigma$ be as above and $x$ be a finite place. Then

(a) $\sigma$ is reachable at $x$ if and only if $I(\sigma)$ is surjective at $x$.

(b) $\sigma$ is observable at $x$ if and only if $O(\sigma)$ is injective at $x$.

Proof. (a) To say that $I(\sigma)$ is surjective at $x$ is equivalent to saying that the morphism $\mathcal{O}^{m} \rightarrow \mathcal{C}$ is surjective at $x$. The assertion follows now from the commutative diagram

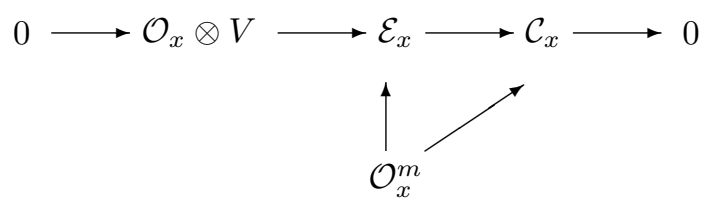

having an exact row.

(b) $\sigma$ is observable at $x$ if and only if the morphism $\left[\theta^{*} C^{*}\right]$ is surjective at $x$. From this, as above, it follows that a necessary and sufficient condition for $\sigma$ to be observable at $x$ is that the homomorphism $\mathcal{O}_{x}^{p} \rightarrow \mathcal{P}_{x}$ be surjective. Since $\mathcal{P}_{x}$ is an $\mathcal{O}_{x}$-module of finite length, this homomorphism gives rise to a homomorphism $\hat{\mathcal{O}}_{x}^{p} \rightarrow \mathcal{P}_{x}$ where ^ denotes the adic completion. Moreover, the surjectivity of the first one is equivalent to that of the second one. Now applying the functor $\operatorname{Hom}\left(\cdot, R / \Omega_{x}\right)$, we complete the proof. (Recall that the above functor is exact, and by the local duality

$$
\operatorname{Hom}\left(\mathcal{P}_{x}, R / \Omega_{x}\right)=\mathcal{S}_{x} \text { and } \operatorname{Hom}\left(R / \Omega_{x}, R / \Omega_{x}\right)=\hat{\mathcal{O}}_{x} .
$$

See [5].)

3. Let us call a function $f \in R$ strictly proper if $\operatorname{ord}_{\infty}(f)>0$.

Definition. A transfer function is a $(p \times m)$-matrix whose entries are strictly proper functions.

A transfer function may be identified with a homomorphism $\mathcal{O}_{\infty}^{m} \rightarrow \mathcal{O}_{\infty}^{p}$ which takes values in $t \mathcal{O}_{\infty}^{p}$.

Let $\sigma=(V, \mathcal{E}, \theta, B, C)$ be a linear system. We have a sequence of $\mathcal{O}_{\infty^{-}}$ homomorphisms

$$
\mathcal{O}_{\infty}^{m} \stackrel{B}{\longrightarrow} \mathcal{E}_{\infty} \stackrel{\theta^{-1}}{\longrightarrow} \mathcal{O}_{\infty} \otimes V \stackrel{C}{\longrightarrow} \mathcal{O}_{\infty}^{p} .
$$

Since $B(\infty): k^{m} \rightarrow \mathcal{E}(\infty)$ is zero, the composed linear map

$$
k^{m} \stackrel{B(\infty)}{\longrightarrow} \mathcal{E}(\infty) \stackrel{\theta^{-1}(\infty)}{\longrightarrow} V \stackrel{C}{\longrightarrow} k^{p}
$$

is also zero. This implies that the above composed homomorphism is a transfer function. We denote it by $T(\sigma)$ and call the transfer function of $\sigma$. 
4. Let $\Sigma$ denote the category of triples $(\mathcal{F}, D, N)$, where $\mathcal{F}$ is a globally generated coherent sheaf of rank $m, D$ is a morphism of $\mathcal{O}^{m}$ into $\mathcal{F}$ such that $D(\infty): k^{m} \rightarrow \mathcal{F}(\infty)$ is bijective, and $N$ is a morphism of $\mathcal{O}^{p}$ into $\mathcal{F}$ such that $N(\infty): k^{p} \rightarrow \mathcal{F}(\infty)$ is zero. Morphisms of this category are defined in the obvious way.

Definition. Let $\sigma=(V, \mathcal{E}, \theta, B, C)$ be a linear system. We let $M H(\sigma)$ denote the cokernel of the morphism

$$
\left[\begin{array}{c}
\theta^{*} \\
B^{*}
\end{array}\right] \longrightarrow \mathcal{O} \otimes V^{*} \oplus \mathcal{O}^{m}
$$

(which is clearly injective) and call it the Martin-Hermann sheaf of $\sigma$. Next, we let $D(\sigma)$ denote the canonical morphism $\mathcal{O}^{m} \rightarrow M H(\sigma)$ and call it the denominator of $\sigma$. Finally, we let $N(\sigma)$ denote the composition of $C^{*}$ and the canonical morphism $\mathcal{O} \oplus V^{*} \rightarrow M H(\sigma)$, and call it the numerator of $\sigma$.

Definition. Let $\sigma$ be a linear system. Put $F R(\sigma)=(M H(\sigma), D(\sigma), N(\sigma))$ and call it the fraction representation of $\sigma$.

It is easily seen that $F R$ is a contravariant functor from the category of linear systems to the category $\Sigma$.

Let $\sigma=(V, \mathcal{E}, \theta, B, C)$ be a linear system and let $F R(\sigma)=(\mathcal{F}, D, N)$.

Proposition 2. The McMillan degree of $\sigma$ is equal to $\operatorname{deg} \mathcal{F}$.

Proof. The proof follows immediately from the exact sequence

$$
0 \longrightarrow \mathcal{E}^{*} \longrightarrow \mathcal{O} \otimes V^{*} \oplus \mathcal{O}^{m} \longrightarrow \mathcal{F} \longrightarrow 0
$$

Proposition 3.The pole sheaf $\mathcal{P}$ of $\sigma$ is canonically isomorphic to coker $D$.

Proof. Dualizing (1) we get an exact sequence

$$
0 \longrightarrow \mathcal{E}^{*} \longrightarrow \mathcal{O} \otimes V^{*} \longrightarrow \mathcal{P} \longrightarrow 0 \text {. }
$$

From this and from (2) follows the statement.

Proposition 4. Let $x$ be a place. Then

(a) $\sigma$ is reachable at $x$ if and only if $\mathcal{F}$ is locally free at $x$.

(b) $\sigma$ is observable at $x$ if and only if the morphism $[D N]$ is surjective at $x$.

Proof. (a) We have an exact sequence

$$
0 \longrightarrow \mathcal{E}_{x}^{*} \longrightarrow \mathcal{O}_{x} \otimes V^{*} \oplus \mathcal{O}_{x}^{m} \longrightarrow \mathcal{F}_{x} \longrightarrow 0
$$

$\sigma$ is reachable at $x$ if and only if the linear map $\mathcal{E}^{*}(x) \rightarrow k(x) \otimes V^{*} \oplus k(x)^{m}$ is injective. Hence, the assertion follows from Proposition 6 of [2], Ch.2, 33 . 
(b) Let $\mathcal{P}$ be the pole sheaf. From the proof of Proposition 1 we know that $\sigma$ is observable at $x$ if and only if $\mathcal{O}_{x}^{p} \rightarrow \mathcal{P}_{x}$ is surjective. So, the assertion follows from the commutative diagram

having an exact row.

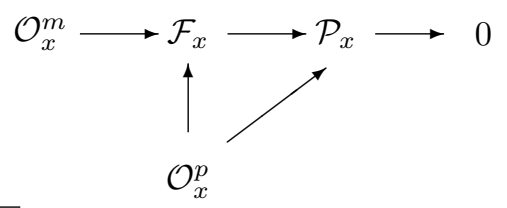

Proposition 5. The transfer function of $\sigma$ is equal to $N_{\infty}^{*} \circ D_{\infty}^{*-1}$.

Proof. Let $L$ denote the canonical morphism $\mathcal{O} \otimes V^{*} \rightarrow \mathcal{F}$. Using the exact sequence

$$
0 \longrightarrow \mathcal{E}_{\infty}^{*} \longrightarrow \mathcal{O}_{\infty} \otimes V^{*} \oplus \mathcal{O}_{\infty}^{m} \longrightarrow \mathcal{F}_{\infty} \longrightarrow 0,
$$

one easily verifies that $D_{\infty} \circ B_{\infty}^{*} \circ \theta^{*-1}=L_{\infty}$. It follows from this that $B_{\infty}^{*} \circ \theta^{*-1} \circ C_{\infty}^{*}=D_{\infty}^{-1} \circ N_{\infty}$.

\section{$\S 2$. Realization Theorem}

Lemma 1. Let $(V, \mathcal{E}, \theta, B, C)$ be a linear system and $\mathcal{F}$ be its MartinHermann sheaf. Then $\operatorname{dim} V \leq \operatorname{dim} H^{0} \mathcal{F}(-1)$.

Proof. By Serre's duality, $H^{0} \mathcal{E}^{*}=H^{1} \mathcal{E}(K)=0$. Hence, from (2) we get an exact sequence

$$
0 \longrightarrow V^{*} \oplus k^{m} \longrightarrow H^{0} \mathcal{F} .
$$

The map $V^{*} \rightarrow H^{0} \mathcal{F}$ must have its image in $H^{0} \mathcal{F}(-1)$ because the composed map $V^{*} \rightarrow H^{0} \mathcal{F} \rightarrow \mathcal{F}(\infty)$ is zero. Consequently, we have a canonical injective linear map $V^{*} \rightarrow H^{0} \mathcal{F}(-1)$. Furthermore, the composition $k^{m} \rightarrow H^{0} \mathcal{F} \rightarrow \mathcal{F}(\infty)$ is bijective.

Lemma 2. Under the notations of the previous lemma the following conditions are equivalent:

(a) $\operatorname{dim} V=\operatorname{dim} H^{0} \mathcal{F}(-1)$;

(b) the map $H^{0} \mathcal{O}(K) \otimes V \oplus H^{0} \mathcal{O}(K)^{m} \rightarrow H^{0} \mathcal{E}(K)$ is surjective;

(c) the canonical sequence $H^{0} \mathcal{O}(K)^{m} \rightarrow H^{0} \mathcal{S} \rightarrow V \rightarrow 0$, where $\mathcal{S}$ is the state sheaf, is exact.

Proof. It follows from the proof of the previous lemma that (a) is equivalent to the bijectivity of the linear map $V^{*} \oplus k^{m} \rightarrow H^{0} \mathcal{F}$. On the other hand, by Serre's duality, (b) is equivalent to the injectivity of the linear map $H^{1} \mathcal{E}^{*} \rightarrow$ $H^{1} \mathcal{O} \otimes V^{*} \oplus H^{1} \mathcal{O}^{m}$. So, the equivalence (a) $\Leftrightarrow$ (b) follows immediately from the cohomological exact sequence

$$
0 \longrightarrow V^{*} \oplus k^{m} \longrightarrow H^{0} \mathcal{F} \longrightarrow H^{1} \mathcal{E}^{*} \longrightarrow H^{1} \mathcal{O} \otimes V^{*} \oplus H^{1} \mathcal{O}^{m},
$$


which can be derived from (2). The equivalence (b) $\Leftrightarrow(\mathrm{c})$ follows immediately from the exact cohomological sequence

$$
0 \longrightarrow H^{0} \mathcal{O}(K) \otimes V \longrightarrow H^{0} \mathcal{E}(K) \longrightarrow H^{0} \mathcal{S} \longrightarrow V \longrightarrow 0
$$

induced by the exact sequence

$$
0 \longrightarrow \mathcal{O}(K) \otimes V \longrightarrow \mathcal{E}(K) \longrightarrow \mathcal{S} \longrightarrow 0 .
$$

In what follows we restrict attention only to linear systems which satisfy the equivalent conditions of the previous lemma, i.e. to linear systems defined as in Introduction.

Theorem 1. The functor FR establishes an equivalence of the category of linear systems with the category $\Sigma^{o p}$.

Proof. Let $(\mathcal{F}, D, N)$ be an object of $\Sigma$. Since $\mathcal{F}$ is generated by global sections, we have, in particular, an exact sequence

$$
0 \longrightarrow H^{0} \mathcal{F}(-1) \longrightarrow H^{0} \mathcal{F} \longrightarrow \mathcal{F}(\infty) \longrightarrow 0 .
$$

Because the composed map

$$
k^{m} \longrightarrow H^{0} \mathcal{F} \longrightarrow \mathcal{F}(\infty)
$$

is bijective, this exact sequence splits canonically, i.e. there is a canonical isomorphism $H^{0} \mathcal{F} \simeq H^{0} \mathcal{F}(-1) \oplus k^{m}$. Furthermore, because $N(\infty)$ is zero, the map $H^{0} N$ takes values in $H^{0} \mathcal{F}(-1)$.

Now put

$\Phi(\mathcal{F}, D, N)=(V, \mathcal{E}, \theta, B, C)$, where $V=\left(H^{0} \mathcal{F}(-1)\right)^{*}, \mathcal{E}=(\operatorname{ker}(\mathcal{O} \otimes$ $\left.\left.H^{0} \mathcal{F} \rightarrow \mathcal{F}\right)\right)^{*}, \theta$ is the canonical morphism $\mathcal{O} \otimes V \rightarrow \mathcal{E}, B$ is the canonical linear map $k^{m} \rightarrow H^{0} \mathcal{E}$ and $C$ is the dual map to $k^{p} \rightarrow H^{0} \mathcal{F}(-1)$. It is easy to see that this is a linear system.

Clearly, $\Phi$ is a contravariant functor from the category $\Sigma$ to the category of linear systems, and one checks without difficulty that $F R$ and $\Phi$ are inverse to each other.

Corollary (Kalman's theorem on realization). The assignment $\sigma \rightarrow T(\sigma)$ induces a bijective correspondence between the isomorphism classes of canonical linear systems and the transfer functions.

(The sense of the word "canonical" is evident.) To prove the corollary we need one lemma.

Let $q=m+p$. Let $\operatorname{Grass}_{m}\left(R^{q}\right)$ be the set of $m$-dimensional subspaces in $R^{q}$ and let $L F Q_{m}\left(\mathcal{O}^{q}\right)$ be the set of locally free quotients of $\mathcal{O}^{q}$ of rank $m$. The elements of $\operatorname{Grass}_{m}\left(R^{q}\right)$ may be identified with the equivalence classes of $(m \times q)$-matrices of rank $m$ with entries in $R$. (Two such matrices $M_{1}$ and $M_{2}$ are equivalent if $M_{2}=G M_{1}$ for some $G \in G L(m, R)$.) The elements of 
$L F Q_{m}\left(\mathcal{O}^{q}\right)$ may be identified with the isomorphic classes of pairs $(\mathcal{F}, f)$, where $\mathcal{F}$ is a vector bundle of rank $m$ and $f$ is an epimorphism of $\mathcal{O}^{q}$ onto $\mathcal{F}$. (Two such pairs $\left(\mathcal{F}_{1}, f_{1}\right)$ and $\left(\mathcal{F}_{2}, f_{2}\right)$ are isomorphic if $f_{2}=\phi \circ f_{1}$ for some isomorphism $\phi: \mathcal{F}_{1} \rightarrow \mathcal{F}_{2}$.)

Lemma 3. There is a natural bijection between the sets $\operatorname{Grass}_{m}\left(R^{q}\right)$ and $L F Q_{m}\left(\mathcal{O}^{q}\right)$.

Proof. Let $F$ be a nondegenerate $(m \times q)$-matrix with elements in $R$ and let $f_{1}, \ldots, f_{q}$ be its columns. Define a vector bundle $\mathcal{F}$ by the formula

$$
\mathcal{F}=\left(R^{m},\left(\sum_{i=1}^{q} \mathcal{O}_{x} f_{i}\right)\right)
$$

Clearly, all $f_{i} \in H^{0} \mathcal{F}$. Hence, we may view the matrix $F$ as a morphism $\mathcal{O}^{q} \rightarrow \mathcal{F}$ (of course, surjective). If now $F^{\prime}=G F$, where $G \in G L(m, R)$, and $\mathcal{F}^{\prime}$ is the corresponding vector bundle, then $G$ clearly defines an isomorphism of $\mathcal{F}$ onto $\mathcal{F}^{\prime}$ such that the diagram

commutes.

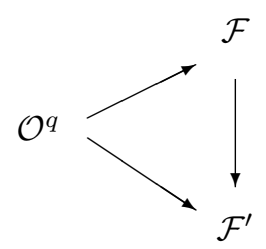

Thus we have a well-defined map from $\operatorname{Grass}_{m}\left(R^{q}\right)$ to $L F Q_{m}\left(\mathcal{O}^{q}\right)$.

Conversely, let $\mathcal{F}=\left(F,\left(F_{x}\right)\right)$ be a vector bundle of rank $m$, and let $f: \mathcal{O}^{q} \rightarrow \mathcal{F}$ be an epimorphism. We then have a surjective $R$-linear map $R^{q} \rightarrow F$. The image of the dual linear map is a linear subspace in $R^{q}$ (= $R^{q \times 1}$ ) of dimension $m$. By the "transposing" of this one we get an element in $\operatorname{Grass}_{m}\left(R^{q}\right)$. It is obvious that if we take a pair isomorphic to $(\mathcal{F}, f)$, we shall come to the same element.

So we have a map from $L F Q_{m}\left(\mathcal{O}^{q}\right)$ into $\operatorname{Grass}_{m}\left(R^{q}\right)$.

It is not hard to verify that the above two maps are inverse to each other.

Proof of the corollary. First note that by

$$
T \longrightarrow\left[I T^{*}\right] \bmod G L(m, R),
$$

where $I$ is the identity $(m \times m)$-matrix, one can identify transfer functions with some elements from $\operatorname{Grass}_{m}\left(R^{q}\right)$.

Now let $T$ be a transfer function. Let $\mathcal{F}$ be the vector bundle corresponding to $\left[I T^{*}\right]$ as in the proof of the previous lemma, and let $D: \mathcal{O}^{m} \rightarrow \mathcal{F}$ and $N: \mathcal{O}^{p} \rightarrow \mathcal{F}$ be the morphisms determined by the matrices $I$ and $T^{*}$, 
respectively. Clearly, $\mathcal{F}_{\infty}=\mathcal{O}_{\infty}^{m}, D_{\infty}=I$ and $N(\infty)=0$. So, $(\mathcal{F}, D, N)$ is a linear system. The proof now can be easily completed.

\section{$\S 3 . \quad$ FEEDBACK}

By a linear system in this section we shall mean a quadruple $(V, \mathcal{E}, \theta, B)$ where $V$ is a linear space over $k$ of finite dimension, $\mathcal{E}$ is a vector bundle over $R$ such that $H^{1} \mathcal{E}(K)=0, \theta$ is a morphism $\mathcal{O} \otimes V \rightarrow \mathcal{E}$ such that the induced map $V \rightarrow \mathcal{E}(\infty)$ is bijective, and $B$ is a linear map $k^{m} \rightarrow H^{0} \mathcal{E}(-1)$. We shall assume that the equivalent conditions of Lemma 2 hold. It follows from the proof of Theorem 1 that such a linear system can be defined as a pair $(\mathcal{F}, f)$ where $\mathcal{F}$ is a globally generated coherent sheaf and $f: \mathcal{O}^{m} \rightarrow \mathcal{F}$ is such that $f(\infty): k^{m} \rightarrow \mathcal{F}(\infty)$ is bijective.

1. Definition. Two linear systems $\left(V_{1}, \mathcal{E}_{1}, \theta_{1}, B_{1}\right)$ and $\left(V_{2}, \mathcal{E}_{2}, \theta_{2}, B_{2}\right)$ are said to be feedback equivalent if there exist an isomorphism $\phi: \mathcal{E}_{2} \rightarrow \mathcal{E}_{1}$, a bijective linear map $\alpha: V_{2} \rightarrow V_{1}$, a linear automorphism $\beta: k^{m} \rightarrow k^{m}$ and a linear map $L: V_{2} \rightarrow k^{m}$ such that

$$
\theta_{2}=\phi^{-1} \theta_{1} \alpha+\phi^{-1} B_{1} L \text { and } B_{2}=\phi^{-1} B_{1} \beta .
$$

Theorem 2 (Martin-Hermann). Two linear systems are feedback equivalent if and only if their Martin-Hermann sheaves are isomorphic.

Proof. Let $\sigma_{1}=\left(V_{1}, \mathcal{E}_{1}, \theta_{1}, B_{1}\right)$ and $\sigma_{2}=\left(V_{2}, \mathcal{E}_{2}, \theta_{2}, B_{2}\right)$ be linear systems, and let $\mathcal{F}_{1}$ and $\mathcal{F}_{2}$ be their Martin-Hermann sheaves, respectively.

Suppose that $\sigma_{1}$ and $\sigma_{2}$ are feedback equivalent. By definition, we then have a commutative diagram

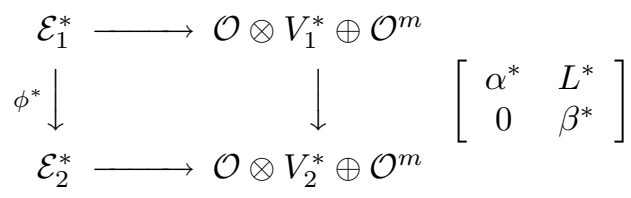

where $\phi, \alpha, \beta$ and $L$ are as above. Since the vertical arrows here are isomorphisms, this diagram yields an isomorphism $\mathcal{F}_{1} \simeq \mathcal{F}_{2}$.

Conversely, suppose that $\mathcal{F}_{1}$ and $\mathcal{F}_{2}$ are isomorphic, and let $\psi$ be any isomorphism of $\mathcal{F}_{1}$ onto $\mathcal{F}_{2}$. We then have a commutative diagram

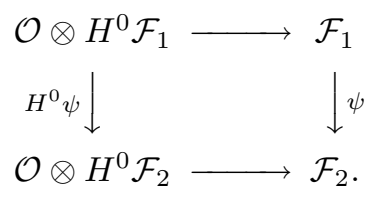

Since $H^{0} \mathcal{F}_{1} \simeq V_{1}^{*} \oplus k^{m}$ and $H^{0} \mathcal{F}_{2} \simeq V_{2}^{*} \oplus k^{m}$, we can find linear maps $\alpha: V_{2} \rightarrow V_{1}, \beta: k^{m} \rightarrow k^{m}, L: V_{2} \rightarrow k^{m}$ and $G: k^{m} \rightarrow V_{1}$ such that 
$\left[\begin{array}{cc}\alpha & G \\ L & \beta\end{array}\right]$ is nonsingular and the following diagram

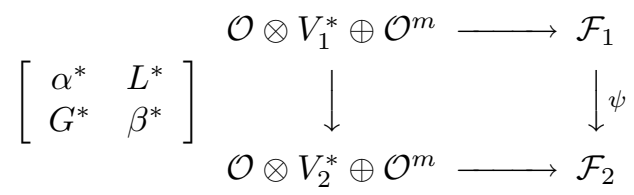

is commutative. This diagram can be extended to the commutative diagram

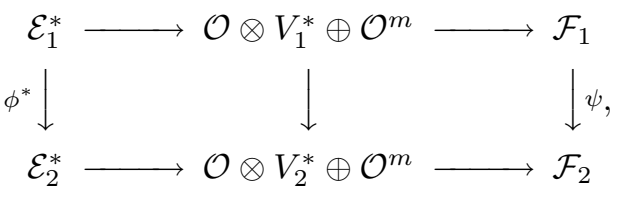

where $\phi$ is an isomorphism of $\mathcal{E}_{2}$ onto $\mathcal{E}_{1}$. We thus have

$$
\left[\begin{array}{ll}
\theta_{1} \alpha+B_{1} L & \theta_{1} G+B_{1} \beta
\end{array}\right]=\left[\begin{array}{l}
\phi \theta_{2} \phi B_{2}
\end{array}\right] .
$$

It remains to show that $G=0$. By the above equality,

$$
\theta_{1}(\infty) G+B_{1}(\infty) \beta=\phi(\infty) B_{2}(\infty) .
$$

Since $B_{1}(\infty)$ and $B_{2}(\infty)$ are zero, we obtain from this that $\theta_{1}(\infty) G=0$; whence $G=0$.

2. Let $\mathcal{F}$ be a globally generated coherent sheaf of rank $m$ which is nonsingular at infinity. Given an effective divisor $D$, which does not contain $\infty$, one can ask whether there exists an injective morphism $f: \mathcal{O}^{m} \rightarrow \mathcal{F}$ such that $\chi(\operatorname{coker} f)=D$. (Note that such a morphism will necessarily be bijective at infinity.) This is the pole placement problem (PPP).

Lemma 4. Let $f: \mathcal{O}^{m} \rightarrow \mathcal{F}$ be an injective morphism, where $\mathcal{F}$ is a coherent sheaf of rank $m$. Let $\mathcal{T}$ be a torsion subsheaf of $\mathcal{F}$ and $\mathcal{F}_{1}=$ $\mathcal{F} / \mathcal{T}$. Then $\chi(\operatorname{coker} f)=\chi\left(\right.$ coker $\left.f_{1}\right)+\chi(\mathcal{T})$, where $f_{1}$ denotes the canonical morphism from $\mathcal{O}^{m}$ to $\mathcal{F}_{1}$.

Proof. We have a commutative diagram

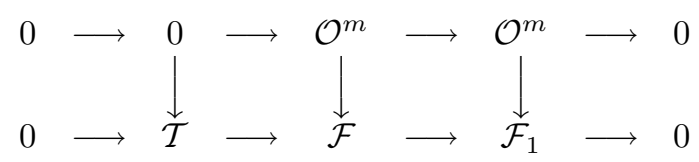

Applying Proposition 2.10 of [1], we get an exact sequence

$$
0 \longrightarrow \mathcal{T} \longrightarrow \operatorname{coker}(f) \longrightarrow \operatorname{coker}\left(f_{1}\right) \longrightarrow 0 .
$$

By Proposition 6.9 of [1] from this follows the lemma. 
The above lemma means in particular that "one cannot change the unreachable modes by feedback". One sees also that it reduces the PPP to the case when $\mathcal{F}$ is a vector bundle.

Observe that if $\mathcal{F}$ is a vector bundle of rank $m$ and $f$ is an injective morphism of $\mathcal{O}^{m}$ into $\mathcal{F}$, then the class of the divisor $\chi$ (coker $f$ ) is equal to the Chern class of $\mathcal{F}$.

Thus, the PPP for a globally generated vector bundle $\mathcal{F}$ of rank $m$ can be posed in the following way: Given an effective divisor $D$ which does not contain $\infty$ and is such that $\operatorname{cl}(D)=\operatorname{ch}(\mathcal{F})$, does there exist an injective morphism $f: \mathcal{O}^{m} \rightarrow \mathcal{F}$ with $\chi(\operatorname{coker} f)=D$ ?

Example. Consider the case $(\star)$. For this case the homomorphism deg: $\mathrm{Cl}(R) \rightarrow \mathbb{Z}$ is an isomorphism. Hence, the Chern class of a vector bundle can be identified with its degree. Next, effective divisors supported in $X-\{\infty\}$ can be identified with monic polynomials in $s$. Let now $\mathcal{F}$ be a vector bundle of rank $m$ and degree $n$. The PPP takes the form: Given a polynomial $P$ in $s$ of degree $n$, choose a morphism $f: \mathcal{O}^{m} \rightarrow \mathcal{F}$ such that $f(\infty)$ is bijective and $\chi(\operatorname{coker} f)=P$. Notice that the sheaf coker $f$ being finite and with support in $X-\{\infty\}$ can be identified with a finite $k[s]$-module or, which is the same thing, with a pair $(V, F)$, where $V$ is a linear space over $k$ and $F$ is an endomorphism of $V$. Clearly, $\chi(\operatorname{coker} f)=$ the characteristic polynomial of $F$.

We do not know if the answer to the PPP is always affirmative. But we have the following

Theorem 3. The PPP has a solution in the case of one input.

Proof. See Proposition 7.7 in [6], Ch.2. Here is another proof. By hypothesis, the sheaves $\mathcal{O}(D)$ and $\mathcal{F}$ are isomorphic. Hence the sheaves $\mathcal{O}$ and $\mathcal{F}(-D)$ also are isomorphic. The sheaf $\mathcal{F}(-D)$ is a subsheaf of $\mathcal{F}$, since $D$ is effective. Thus, there exists a monomorphism $f: \mathcal{O} \rightarrow \mathcal{F}$ whose image is $\mathcal{F}(-D)$. We have: coker $f=\mathcal{F} / \mathcal{F}(-D)$. Because $\mathcal{F}$ is locally free of rank 1 , it follows that coker $f \simeq \mathcal{O} / \mathcal{O}(-D)$; whence $\chi(\operatorname{coker} f)=D$.

The following lemma may be helpful when one attempts to solve the PPP.

Lemma 5. Let $\mathcal{F}$ be a globally generated vector bundle of rank $m$. Let $\mathcal{O} \rightarrow \mathcal{F}$ be an injective morphism such that its cokernel $\mathcal{F}_{1}$ is a vector bundle too. (Such a morphism always exists.) Assume that the canonical map $H^{0} \mathcal{F} \rightarrow H^{0} \mathcal{F}_{1}$ is surjective. If the $\mathrm{PPP}$ is solvable for $\mathcal{F}_{1}$, then it is solvable for $\mathcal{F}$ as well.

Proof. Let $D$ be an effective divisor such that $\infty \notin \operatorname{Supp} D$ and $\operatorname{cl}(D)=$ $\operatorname{ch}(\mathcal{F})$. Clearly, $\operatorname{ch}\left(\mathcal{F}_{1}\right)=\operatorname{ch}(\mathcal{F})$. According to our assumption there exists an injective morphism $f_{1}: \mathcal{O}^{m-1} \rightarrow \mathcal{F}_{1}$ such that $\chi\left(\right.$ coker $\left.f_{1}\right)=D$. Since 
$H^{0} \mathcal{F} \rightarrow H^{0} \mathcal{F}_{1}$ is surjective, $f$ can be lifted to some $f: \mathcal{O}^{m} \rightarrow \mathcal{F}$. We then have a commutative diagram

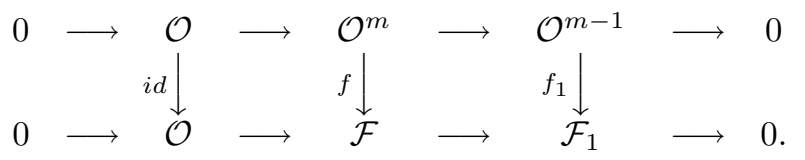

One can derive easily from it an isomorphism coker $f \simeq \operatorname{coker} f_{1}$.

Remark. From the above lemma one can deduce at once the classical result on state feedback. Indeed, let $g=0$. Let $\mathcal{O} \rightarrow \mathcal{F}$ be any injective morphism with a locally free quotient $\mathcal{F}_{1}$. We then have an exact sequence

$$
0 \longrightarrow \mathcal{O} \longrightarrow \mathcal{F} \longrightarrow \mathcal{F}_{1} \longrightarrow 0
$$

which yields an exact sequence of cohomologies

$$
H^{0} \mathcal{F} \longrightarrow H^{0} \mathcal{F}_{1} \longrightarrow H^{1} \mathcal{O} .
$$

Since $H^{1} \mathcal{O}=0$, we find that the additional condition of Lemma 6 holds automatically. By the induction argument we obtain the desired result.

\section{APPENDIX}

Here we give a brief review of the theory of algebraic function fields (in one variable). For additional information, see $[3,4,6,11,12]$. (Recall that algebraic function fields are equivalent as objects to nonsingular complete irreducible algebraic curves.)

In what follows, $k$ is a ground field.

An algebraic function field over $k$ is a finitely generated extension of $k$ of transcendence degree 1 or, which is the same thing, a finite extension of a field isomorphic to the rational function field over $k$ in one indeterminate.

Let $R$ be such a field. For simplicity assume that it is separable over $k$.

A place of $R$ is an equivalence class of nontrivial absolute values of $R$ trivial on $k$. (Recall that two nontrivial absolute values ||$_{1}$ and ||$_{2}$ of a field are said to be equivalent if they induce the same topology. It is not hard to prove that this holds if and only if ||$_{2}=||_{1}^{\lambda}$ for some $\lambda>$ 0 . See [8], Ch.XII, Prop.1.) Denote the set of all places by $X$. There is a one-to-one correspondence between the places of $R$ and the discrete valuations of $R$ trivial to $k$. A discrete valuation corresponding to a place $x$ is denoted by $\operatorname{ord}_{x}$.

A function $f \in R$ is said to be regular at a place $x$ if $\operatorname{ord}_{x}(f) \geq 0$. The set of regular functions at $x$, denoted by $\mathcal{O}_{x}$, is a discrete valuation ring. The residue field $k(x)$ of $\mathcal{O}_{x}$ is a finite extension of $k$; one denotes its degree by $d(x)$. A place $x$ is said to be rational if $d(x)=1$. An affine set is a complement to a nonempty finite set of places of $X$. If $U$ is an 
affine set, then the ring of regular functions on $U$ is a Dedekind domain. Its maximal ideals are in a natural one-to-one correspondence with the places in $U$. The affine sets together with the empty set and the whole space form a topology on $X$. A constant is a rational function which is algebraic over $k$ or, equivalently, which is regular everywhere. The constants form a finite extension of $k$. If $R$ possesses at least one rational place, then the constant field coincides with $k$.

A divisor is an element of the free abelian group $\operatorname{Div}(R)$ generated by places. There is an evident partial order on divisors. One says that a divisor $D$ is effective if $D \geq 0$. If $f$ is a rational function $\neq 0$, then $\operatorname{ord}_{x}(f)=0$ for almost all $x$, and therefore $[f]=\sum \operatorname{ord}_{x}(f) x$ is a divisor. It is called the principal divisor belonging to $f$. The quotient group of $\operatorname{Div}(R)$ modulo the principal divisors is called the divisor class group and is denoted by $\mathrm{Cl}(R)$. For any divisor $D=\sum n(x) x$ one puts $\operatorname{deg} D=\sum n(x) d(x)$. Clearly, $\operatorname{deg}: \operatorname{Div}(R) \rightarrow \mathbb{Z}$ is a homomorphism. An important fact is that the degree of a principal divisor is zero. This makes possible to define deg : $\mathrm{Cl}(R) \rightarrow \mathbb{Z}$.

The space of differential forms of $R$ over $k$ is a "universal" $R$-linear space $\Omega(R / k)$ equipped with a $k$-linear map $d: R \rightarrow \Omega(R / k)$ satisfying the condition $d(f g)=f d g+g d f ; f, g \in R$. Since $R / k$ is a finitely generated separable extension of transcendence degree 1 , this is a linear space of dimension 1.

Let $\omega$ be a nonzero differential form. If $x$ is a place and if $\pi$ is a uniformizer at $x$, then $\omega=f d \pi$ for some $f \in R$. Put $\operatorname{ord}_{x}(\omega)=\operatorname{ord}_{x}(f)$. This definition does not depend on choosing $\pi$. For all but finitely many places $x$ one has: $\operatorname{ord}_{x}(\omega)=0$. Therefore the formal sum $[\omega]=\sum \operatorname{ord}_{x}(\omega) x$ is a divisor. It is called the divisor associated to $\omega$.

A vector bundle $\mathcal{E}=\left(E,\left(E_{x}\right)\right)$ of rank $r$ consists of a linear space $E$ over $R$ of dimension $r$ and of a 'coherent' system $\left(E_{x}\right)$ of $\mathcal{O}_{x}$-lattices in $E$ (i.e. of free $\mathcal{O}_{x}$-submodules of $E$ of maximal rank). The coherence means that if $\left(e_{1}, \ldots, e_{r}\right)$ is a basis of $E$, then $E_{x}=\mathcal{O}_{x} e_{1}+\cdots+\mathcal{O}_{x} e_{r}$ for almost every $x$. (See [12], Ch.6.) The elements of $E$ are called the rational sections of $\mathcal{E}$. The elements of $\Gamma(\mathcal{E})=\cap E_{x}$ are called the global sections. The simplest example of a vector bundle is $\mathcal{O}=\left(R,\left(\mathcal{O}_{x}\right)\right)$.

A morphism of a vector bundle $\left(E,\left(E_{x}\right)\right)$ into a vector bundle $\left(F,\left(F_{x}\right)\right.$ is a linear map $\theta: E \rightarrow F$ over $R$ such that $\theta\left(E_{x}\right) \subseteq F_{x}$ for each $x$.

Let $\mathcal{E}=\left(E,\left(E_{x}\right)\right)$ be a vector bundle of rank $r$. Let $e_{1}, \ldots, e_{r}$ be linearly independent rational sections of $\mathcal{E}$. For each place $x$ choose any basis $\left(e_{x 1}, \ldots, e_{x r}\right)$ of $E_{x}$ and put $\left[e_{1}, \ldots, e_{r}\right]_{x}=\operatorname{ord}_{x}\left(\operatorname{det}\left(a_{i j}\right)\right)$, where $a_{i j} \in R$ are defined by $e_{x i}=\sum a_{i j} e_{x j}$. This is an integer which does not depend on choosing $\left(e_{x 1}, \ldots, e_{x r}\right)$. Clearly, $\left[e_{1}, \ldots, e_{r}\right]_{x}=0$ for almost every $x$. Therefore $\left[e_{1}, \ldots, e_{r}\right]=\sum\left[e_{1}, \ldots, e_{r}\right]_{x} \cdot x$ is a divisor. The class of this divisor is independent of $e_{1}, \ldots, e_{r}$. This is the Chern class of $\mathcal{E}$ denoted by $\operatorname{ch}(\mathcal{E})$. 
A vector bundle of rank 1 is called a linear bundle. If $D=\sum n_{x} \cdot x$ is a divisor, we have a linear bundle $\mathcal{O}(D)=\left(R,\left(\left\{f \mid \operatorname{ord}_{x} f \geq-n_{x}\right\}\right)\right)$. Every linear bundle is isomorphic to $\mathcal{O}(D)$ for some $D$. Note that if $\mathcal{L}$ is a linear bundle, then $\mathcal{L} \simeq \mathcal{O}(D)$ if and only if $\operatorname{ch}(\mathcal{L})=\operatorname{cl}(D)$.

A finite sheaf is a collection of $\mathcal{O}_{x}$-modules $M_{x}$ of finite length such that $M_{x}=0$ for almost all $x$. The characteristic divisor of a finite sheaf $\mathcal{M}=\left(M_{x}\right)$ denoted by $\chi(\mathcal{M})$ is defined as the divisor $\sum$ length $\left(M_{x}\right) x$.

We are going now to define sheaves and their cohomologies. We shall do this under the hypothesis that we are given a fixed nonconstant rational function $s$.

Let $U_{1}$ and $U_{2}$ be the sets where $s$ and $s^{-1}$ are respectively regular. These are affine sets, and they cover the whole of $X$. Denote their intersection by $U$ and put: $A_{1}=\mathcal{O}(U), A_{2}=\mathcal{O}\left(U_{2}\right)$ and $A=\mathcal{O}(U)$. A sheaf is a quintuple $\left(M_{1}, M_{2}, M, r_{1}, r_{2}\right)$ where $M_{1}, M_{2}$ and $M$ are modules over the rings $A_{1}, A_{2}$ and $A$, respectively, and $r_{1}: M_{1} \rightarrow M$ and $r_{2}: M_{2} \rightarrow M$ are homomorphisms over $A_{1}$ and $A_{2}$, respectively. It is required that the canonical homomorphisms

$$
A \otimes_{A_{1}} M_{1} \longrightarrow M \quad \text { and } \quad A \otimes_{A_{2}} M_{2} \longrightarrow M
$$

be isomorphisms. Here are

Examples. 1) Let $\left(E,\left(E_{x}\right)\right)$ be a vector bundle. Then

$$
\left(\bigcap_{x \in U_{1}} E_{x}, \bigcap_{x \in U_{2}} E_{x}, \bigcap_{x \in U} E_{x}, j_{1}, j_{2}\right),
$$

where $j_{1}$ and $j_{2}$ are the canonical inclusions, is a sheaf.

2) Let $\left(M_{x}\right)$ be a finite sheaf. Then

$$
\left(\underset{x \in U_{1}}{\oplus} M_{x}, \underset{x \in U_{2}}{\oplus} M_{x}, \underset{x \in U}{\oplus} M_{x}, r_{1}, r_{2}\right),
$$

where $r_{1}$ and $r_{2}$ are the obvious restriction maps, is a sheaf.

$3)$ Let $E$ be a linear space over $R$. Then $(E, E, E, i d, i d)$ is a sheaf. We shall denote it simply by $E$.

A sheaf is said to be coherent if the modules $M_{1}$ and $M_{2}$ are of finite type. It is said to be locally free if these modules are projective, and is said to be torsion if they are torsion modules. One can identify coherent locally free sheaves with vector bundles (see Example 1)), and coherent torsion sheaves with finite sheaves (see Example 2)).

For each sheaf $\mathcal{F}$ one defines in the obvious way the space of global sections $\Gamma(\mathcal{F})$, the stalk $\mathcal{F}_{x}$ and the reduced stalk $\mathcal{F}(x)$ at a point $x$, the support $\operatorname{Supp} \mathcal{F}$ and the $\operatorname{rank} \operatorname{rk}(\mathcal{F})$. (See, for example, [9], §1.1.) One defines in the standard way subsheaves and quotient sheaves, morphisms, kernels, cokernels and images of morphisms, various operations on sheaves (direct sums, direct limits, tensor products, sheaves Hom, dual sheaves), 
exact sequences of sheaves. For a sheaf $\mathcal{F}$ and a divisor $D$ one usually writes $\mathcal{F}(D)$ for $\mathcal{F} \otimes \mathcal{O}(D)$. One defines (as in [9], §1.1, for example) the functors $\mathcal{E} x t^{i}(\cdot, \mathcal{O}), i=0,1$.

If $V$ is a linear space $V$ over $k$ of finite dimension and $\mathcal{F}$ is a coherent sheaf, then $\operatorname{Hom}(\mathcal{O} \otimes V, \mathcal{F})=\operatorname{Hom}(V, \Gamma(\mathcal{F}))$. In particular, one has a canonical morphism $\mathcal{O} \otimes \Gamma(\mathcal{F}) \rightarrow \mathcal{F}$. If this morphism is surjective, one says that $\mathcal{F}$ is generated by global sections.

If $\phi: \mathcal{F} \rightarrow \mathcal{G}$ is a morphism, then for each point $x$ one has a homomorphism $\phi_{x}: \mathcal{F}_{x} \rightarrow \mathcal{G}_{x}$ and a linear map $\phi(x): \mathcal{F}(x) \rightarrow \mathcal{G}(x)$.

For each sheaf $\mathcal{F}=\left(M_{1}, M_{2}, M, r_{1}, r_{2}\right)$ we introduce $k$-linear spaces $C^{0} \mathcal{F}=M_{1} \oplus M_{2}$ and $C^{1} \mathcal{F}=M$, and define $k$-linear map $d: C^{0} \mathcal{F} \rightarrow C^{1} \mathcal{F}$ by the formula $d\left(m_{1}, m_{2}\right)=r_{1}\left(m_{1}\right)-r_{2}\left(m_{2}\right)$. We denote the kernel and cokernel of this map by $H^{0} \mathcal{F}$ and $H^{1} \mathcal{F}$, respectively, and call them the 0 -dimensional and 1-dimensional cohomology spaces of $\mathcal{F}$, respectively.

Clearly, $H^{0}$ and $H^{1}$ are functors. Here are their principal properties:

(a) For each exact sequence of sheaves $0 \rightarrow \mathcal{F}_{1} \rightarrow \mathcal{F} \rightarrow \mathcal{F}_{2} \rightarrow 0$ there is an exact sequence of cohomologies

$$
0 \longrightarrow H^{0} \mathcal{F}_{1} \longrightarrow H^{0} \mathcal{F} \longrightarrow H^{0} \mathcal{F}_{2} \longrightarrow H^{1} \mathcal{F}_{1} \longrightarrow H^{1} \mathcal{F} \longrightarrow H^{1} \mathcal{F}_{2} \longrightarrow 0
$$

(Moreover, this cohomological sequence is functorial).

(b) $H^{0}$ and $H^{1}$ commute with direct limits;

(c) $H^{0} \mathcal{F}=\Gamma(\mathcal{F})$ for each $\mathcal{F}$;

(d) $H^{1} \mathcal{F}=0$ for each finite $\mathcal{F}$;

(e) $H^{1} R=0$.

(a) follows from Proposition 2.10 of [1]. Other properties are obvious. It is not difficult to prove that the above properties determine $H^{0}$ and $H^{1}$ uniquely.

The following is the basic result on cohomologies.

Finiteness theorem. If $\mathcal{F}$ is a coherent sheaf, then $h^{i} \mathcal{F}=\operatorname{dim} H^{i} \mathcal{F}<$ $+\infty$.

The genus of the curve is the number $g=h^{1} \mathcal{O}$.

The degree of a coherent sheaf $\mathcal{F}$ is defined by the formula $\operatorname{deg} \mathcal{F}=$ $h^{0} \mathcal{F}-h^{1} \mathcal{F}-\operatorname{rk}(\mathcal{F})(1-g)$. The degree is an additive function. This means that if $0 \rightarrow \mathcal{F}_{1} \rightarrow \mathcal{F} \rightarrow \mathcal{F}_{2} \rightarrow 0$ is an exact sequence of coherent sheaves, then $\operatorname{deg} \mathcal{F}=\operatorname{deg} \mathcal{F}_{1}+\operatorname{deg} \mathcal{F}_{2}$.

Note that if $\mathcal{M}$ is a finite sheaf, then $\operatorname{deg} \mathcal{M}=\operatorname{deg} \chi(\mathcal{M})$.

For vector bundles we have the famous

Riemann-Roch theorem. If $\mathcal{E}$ is a vector bundle, then $\operatorname{deg} \mathcal{E}=$ $\operatorname{deg} \operatorname{ch}(\mathcal{E})$.

Let $K$ be the divisor of the differential $d s$. If $\mathcal{E}$ is a vector bundle, set $\check{\mathcal{E}}=\mathcal{E}^{*}(K)$. Clearly, $\check{\mathcal{E}}=\mathcal{E}$. We finish with the following important result. 
Serre's duality theorem. For every vector bundle $\mathcal{E}$ there is a nondegenerate canonical pairing $H^{0} \check{\mathcal{E}} \times H^{1} \mathcal{E} \rightarrow k$.

\section{REFERENCES}

1. M.F. Atiyah and I.G. McDonald, Introduction to commutative algebra. Addison-Wesley, Reading, MA, 1969.

2. N. Bourbaki, Algébre commutative. Éléments de Mathématique, Chap. 1-7. Hermann, Paris, 1961-1965.

3. C. Chevalley, Introduction to the theory of algebraic functions of one variable. American Mathematical Society, New York, 1951.

4. H. Grauert and R. Remmert, Theorie der Steinschen Räume. SpringerVerlag, New York, 1977.

5. A. Grothendieck, Local cohomology. Lecture Notes in Mathematics, v.41. Springer-Verlag, Berlin, 1967.

6. R. Hartshorne, Algebraic geometry. Graduate Texts in Mathematics, v.52. Springer-Verlag, New York, 1977.

7. R. Hermann, Topics in the geometric theory of linear systems. Interdisciplinary Mathematics, v.22. Math. Sci. Press, Brookline, MA, 1985.

8. S. Lang, Algebra. Addison-Wesley, Reading, MA, 1965.

9. V.G. Lomadze, Finite-dimensional time invariant linear dynamical systems: Algebraic theory. Acta Applicandae Mathematicae 19 (1990), 149201.

10. V.G. Lomadze, Linear systems and coherent sheaves. (Russian) Bull. Acad. Sci. Georgian SSR, 126(1987), No. 1, 37-40.

11. J.P. Serre, Groupes algebriques et corps de classes. Hermann, Paris, 1969.

12. A. Weil, Basic number theory. Grundlehren der Mathematischen Wissenschaften, Bd. 144. Springer-Verlag, New York, 1967.

(Received 16.07.1993)

Author's address:

A. Razmadze Mathematical Institute

Georgian Academy of Sciences

1, Z. Rukhadze St., Tbilisi 380093

Republic of Georgia 